\title{
Pharmacological Targeting of Long QT Mutant Sodium Channels
}

D.W. Wang, K. Yazawa, N. Makita, A.L. George, Jr., and P.B. Bennett

Department of Pharmacology and Department of Medicine, Vanderbilt University School of Medicine, Nashville, Tennessee 37232-6602

\begin{abstract}
The congenital long QT syndrome (LQTS) is an inherited disorder characterized by a delay in cardiac cellular repolarization leading to cardiac arrhythmias and sudden death often in young people. One form of the disease (LQT3) involves mutations in the voltage-gated cardiac sodium channel. The potential for targeted suppression of the LQT defect was explored by heterologous expression of mutant channels in cultured human cells. Kinetic and steady state analysis revealed an enhanced apparent affinity for the predominantly charged, primary amine compound, mexiletine. The affinity of the mutant channels in the inactivated state was similar to the wild type (WT) channels $\left(\mathrm{IC}_{50} \sim 15-20 \mu \mathrm{M}\right)$, but the late-opening channels were inhibited at significantly lower concentrations $\left(\mathrm{IC}_{50}=2-3 \mu \mathrm{M}\right)$ causing a preferential suppression of the late openings. The targeting of the defective behavior of the mutant channels has important implications for therapeutic intervention in this disease. The results provide insights for the selective suppression of the mutant phenotype by very low concentrations of drug and indicate that mexiletine equally suppresses the defect in all three known LQT3 mutants. (J. Clin. Invest. 1997. 99:17141720.) Key words: SCN5A • human heart • hH1 • LQT3 • $\mathrm{Na}^{+}$channel $\bullet$ antiarrhythmic agent $\bullet$ mexiletine
\end{abstract}

\section{Introduction}

The congenital long QT syndrome (LQTS) ${ }^{1}$ is a myocardial disease caused by mutations at distinct genetic loci $(1,2)$. One form of the disease (LQT3) is caused by mutations in the human voltage-gated cardiac sodium channel gene (SCN5A) (3-6). Three different mutations in SCN5A were identified in subsets of long QT syndrome patients $(5,6)$. These include an inframe deletion of three amino acids (lys 1,505, proline 1,506, and glutamine 1,507, $\Delta \mathrm{KPQ})$, and two point mutations: N1325S (asparagine 1,325 converted to serine) and R1644H (arginine 1,644 converted to histidine). The functional defects caused by these sodium channel mutations on gating have re-

Address correspondence to Paul B. Bennett, Ph.D., Department of Pharmacology, 558 MRB II, Vanderbilt University School of Medicine, Nashville, TN 37232-6602. Phone: 615-936-1683; FAX: 615-3224707; E-mail: Paul.Bennett@mcmail.vanderbilt.edu

Received for publication 3 September 1996 and accepted in revised form 21 January 1997.

1. Abbreviations used in this paper: LQT, long QT; WT, wild type.

J. Clin. Invest.

(c) The American Society for Clinical Investigation, Inc. 0021-9738/97/04/1714/07 \$2.00

Volume 99, Number 7, April 1997, 1714-1720 cently been described (7-9). The mutations cause the normally transiently opening sodium channels to continue to reopen at depolarized membrane potentials giving rise to a sustained inward current which prolongs the cardiac action potential.

An important goal for rational treatment of this disorder is to define the molecular basis and conditions for suppression of this defect (10). One strategy is to target and selectively suppress the phenotype produced by the mutation. A recent study suggests that this may be possible. An et al. (11) demonstrated suppression of late-opening sodium channels by high concentrations of lidocaine in the $\triangle \mathrm{KPQ}$ mutant channels. However, lidocaine may not be the optimal drug for this purpose because its mechanism of action is to block inactivated rather than open sodium channels $(12,13)$ and it must be given intravenously to patients. Dumaine et al. (8) have recently reported that there are multiple distinct mechanisms for the three LQT3 mutant channels and that high concentrations of mexiletine could inhibit these mutant channels when expressed in Xenopus laevis oocytes. However, the range of therapeutic plasma concentrations of mexiletine for treatment of arrhythmias is much lower than they tested $(4-11 \mu \mathrm{M})$, and $X$. laevis oocytes can be problematic for studies of drug effects possibly due to their large lipophilic yolk. Thus, it is unknown whether therapeutic levels of an antiarrhythmic agent can affect these channels, how the inhibition compares to wild type (WT) channels, or whether such a drug will affect each of the mutant channels differently.

Mexiletine is an orally available antiarrhythmic agent which like lidocaine inhibits sodium channels $(4,12-27)$. The charged form interacts with the open state and the uncharged form interacts with the inactivated state of the channel (14-17). The primary mechanism of inhibition of $\mathrm{WT} \mathrm{Na}^{+}$channels by mexiletine involves the uncharged drug interacting with the inactivated state of the channel $(25,26,28)$. The open state is normally too brief to permit significant interactions with mexiletine $(13,22,29-32)$. Nevertheless, the pKa of mexiletine is 9.3 , therefore, $99 \%$ of the drug is charged at physiological $\mathrm{pH}$. Thus, under normal conditions, only $1 \%$ of mexiletine is in the form that blocks inactivated channels. We reasoned that the major charged species of mexiletine may opportunistically suppress the abnormal repetitive reopenings of sodium channels in LQT3.

To test this hypothesis, LQT3 sodium channel mutations were engineered in the recombinant human heart sodium channel (hH1) by site-directed mutagenesis (9). WT and mutant channels were expressed heterologously in human embryonic kidney cells. Channel behavior and inhibition by mexiletine were investigated by whole-cell patch clamp methods. Voltage clamp protocols were used to favor occupancy of distinct conformational states of the antiarrhythmic drug receptor (i.e., primarily rested or primarily inactivated). Remarkably, the results show that LQT3 mutant channels are much more sensitive to inhibition by mexiletine than are WT sodium channels. The defective late openings are selectively suppressed more than the peak sodium current and these late openings 
can be suppressed by concentrations at the lower end of the therapeutic range. Furthermore, the defect is suppressed in each of the three mutant channels to approximately the same extent indicating that mexiletine may be useful for treatment of this disorder regardless of the specific sodium channel mutation.

\section{Methods}

Voltage clamp methods. The methods used have been described previously $(9,27,32,33)$. Sodium currents were recorded using the whole-cell patch clamp technique (34). Electrodes resistances ranged from $0.8-2 \mathrm{M} \Omega$. Voltage clamp command pulses were generated using pCLAMP software (v6.03; Axon Instruments, Inc., Foster City, $\mathrm{CA})$. Currents were filtered at $5 \mathrm{kHz}(-3 \mathrm{~dB}$, 4-pole Bessel filter). An Axopatch 200 patch clamp amplifier was used with series resistance compensation (Axon Instruments, Inc.). The holding potential for all pulse protocols was $-120 \mathrm{mV}$. Experiments were done at room temperature $\left(20-22^{\circ} \mathrm{C}\right)$. The bath solution contained: $145 \mathrm{mM} \mathrm{NaCl} ; 4$ $\mathrm{mM} \mathrm{KCl} ; 1.8 \mathrm{nM} \mathrm{CaCl}_{2} ; 1.0 \mathrm{mM} \mathrm{MgCl} ; 10 \mathrm{mM}$ Hepes; and $10 \mathrm{mM}$ glucose, $\mathrm{pH}$ 7.35. The pipette solution contained (intracellular solution): $10 \mathrm{mM} \mathrm{NaF} ; 110 \mathrm{nM} \mathrm{CsF} ; 20 \mathrm{nM} \mathrm{CsCl} ; 10 \mathrm{nM}$ EGTA ; and 10 $\mathrm{nM}$ Hepes, $\mathrm{pH}$ 7.35. Data are presented as mean \pm SEM.

Site-directed mutagenesis of human heart sodium channel (3) was performed as described $(7,9)$. Mutant and WT cDNAs were subcloned into pRc/CMV (Invitrogen Corp., San Diego, CA) for expression in mammalian cells $(9,27,33)$. Multiple independent recombinants were sequenced thoroughly in the mutated region and and tested to confirm expression and identical behavior. At least three independent clones were tested for expression studies.

The calcium phosphate method was used to express mutant and WT human heart sodium channel in a transformed human kidney cell line (HEK 293t, also called tsA-201) stably expressing the SV40 T-antigen. Transfections used $10 \mu \mathrm{g}$ of channel encoding plasmid DNA and $2 \mu \mathrm{g}$ of plasmid encoding green fluorescent protein to visually identify cells expressing exogenous channel DNA.

Experimental protocols and data analysis. Voltage clamp pulse protocols were designed to measure both peak and late-opening sodium channels. To characterize late-opening channels, the variance of the channel gating fluctuations was measured. Because the macroscopic current measured in whole-cell recordings is the ensemble average behavior of all channels in the cell membrane, there is a variance associated with the sodium current that reflects the random nature of the underlying single channel events (28). The ensemble variance, $<\mathrm{VAR}>$, is related to channel gating according to the following relationship:

$<V A R>=P(1-P) N i^{2}$

where $\mathrm{P}$ is the open probability, $\mathrm{N}$ is the number of channels, and $i$ is the single-channel current. The term $\mathrm{P}(1-\mathrm{P})$ contains all of the information about channel gating and block. This term is amplified by two constants, $\mathrm{N}$ (the number of channels), and $\mathrm{i}^{2}$ (the single-channel current squared). Thus, the variance is an exquisitely sensitive measure of gating and block.

Previous studies (35) indicate that inhibition of sodium channels by local anesthetic/antiarrhythmic type agents such as mexiletine likely occurs when a single molecule of the agent binds to a single channel. The binding can be approximated by an interaction with a single site. We assume that a drug-bound channel is nonconducting. Therefore, block of channels can be defined as

Block $=1-\left(I / I_{\max }\right)=[M] /\left([M]+K_{\mathrm{D}}\right)$

and

$K_{\mathrm{D}}=([M] /$ Block $)-[M]$ where $I_{\max }$ is the unblocked current in the absence of mexiletine (M) and $\mathrm{I}$ is the magnitude of the current in the presence of a given concentration ([M]) of the drug. Alternatively, Eq. 2 can be fitted to concentration-block data to estimate the apparent $K_{\mathrm{D}}\left(\mathrm{IC}_{50}\right)$. Assuming a first-order binding interaction between the drug and the channel, the time constant for block development should depend on the mexiletine concentration ([M]) according to the following relationship where $k$ and 1 represent the association and dissociation rate constants, respectively:

$\tau_{\text {Block }}=([M] k+1)^{-1}$.
A

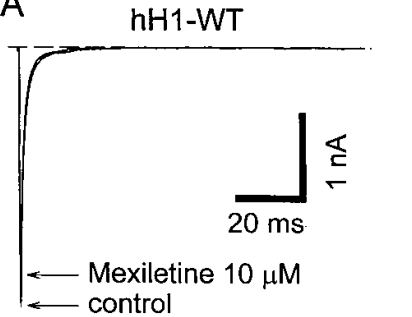

C

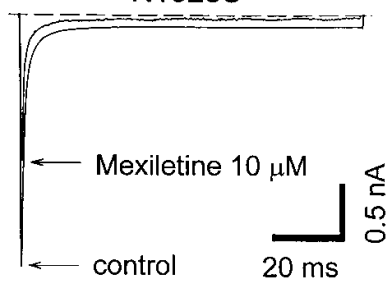

$\mathrm{E}$

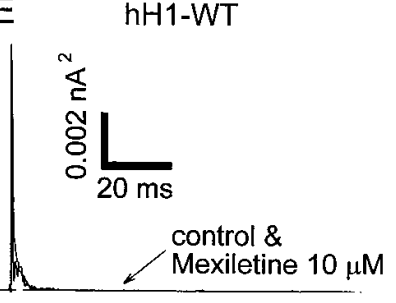

G

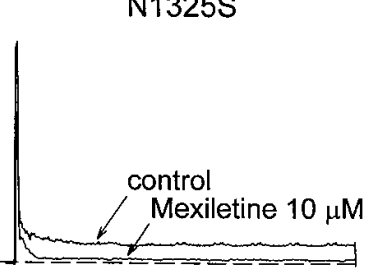

$\mathrm{B}$

$\mathrm{D}$

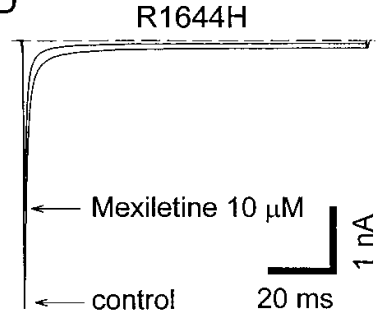

$\mathrm{F}$

$\triangle K P Q$

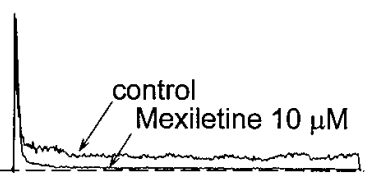

$\mathrm{H}$

\section{$\mathrm{R} 1644 \mathrm{H}$}

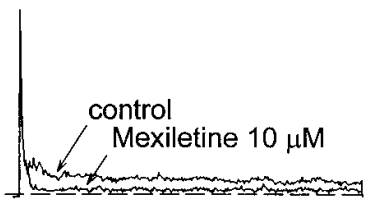

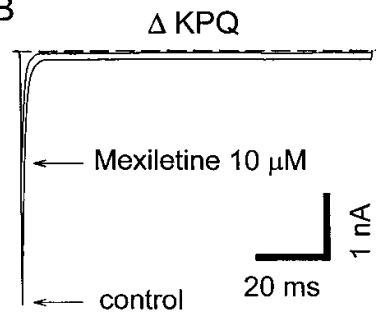

Figure 1. Whole-cell recording of WT, $\triangle \mathrm{KPQ}, \mathrm{N} 1325 \mathrm{~S}$, and R1644H human heart $\mathrm{Na}^{+}$channels expressed in human kidney (HEK293t) cells. Voltage clamp steps of $100-\mathrm{ms}$ duration to $-20 \mathrm{mV}$ from a holding potential of $-120 \mathrm{mV}$ were applied every $5 \mathrm{~s}$. $(A-D)$ Inward sodium current $\left(\mathrm{I}_{\mathrm{Na}}\right)$ and the suppression of peak and late $\mathrm{I}_{\mathrm{Na}}$ by mexiletine $(10 \mu \mathrm{M})$. (Arrows) The level of the peak $\mathrm{I}_{\mathrm{Na}}$ in the presence of mexiletine. The late sodium current of the mutant channels was reduced by mexiletine in all cases. $(E-H)$ Ensemble variance of the $\mathrm{Na}^{+}$ channel gating fluctuations. The average of 20 consecutive recordings is shown along with the variance computed during the averaging process. The nonstationary variance is a sensitive indicator of gating. Note the nonzero variance that occurs after $20 \mathrm{~ms}$ in the mutant channels. As channels inactivate or are blocked, the gating variance and current decrease. 
Although these assumptions oversimplify the actual interactions, they provide a tractable way to characterize the dominant binding and unbinding reactions.

Block of sodium channels by mexiletine was estimated in two different ways. First, sodium current was measured at $-20 \mathrm{mV}$ after holding the cell membrane potential at $-120 \mathrm{mV}$ for $5 \mathrm{~s}$ to remove inactivation. This procedure was repeated every $5 \mathrm{~s}$ and sodium currents were recorded before and during bath application of mexiletine. From these recordings, the ensemble average current and variance was calculated. This analysis will detect both interactions with rested and/or open channel conformations. The second protocol was designed to measure interactions of mexiletine with the inactivated state of the channel. In this case, a dual-pulse protocol was employed. The first (prepulse) was used to inactivate the channels for various periods of time. This was followed by a brief step ( $300 \mathrm{~ms})$ to -120 $\mathrm{mV}$ to allow recovery of inactivated but unblocked channels, followed by a test step to $-20 \mathrm{mV}$ to assess the fraction of unblocked channels. From this measurement and by knowing the magnitude of fully unblocked current, the fraction of channels blocked by a given concentration of mexiletine was determined. The time course of block by mexiletine was estimated by varying the duration of the prepulse. The ratios were made of the sodium current in mexiletine divided by the control current measured after the same duration prepulse in the same cell. These ratios were averaged and plotted as a function of prepulse potential to give the time course of block (see Fig. 3).

\section{Results}

Mexiletine inhibits mutant sodium channels. Fig. 1 demonstrates the phenotype of the three LQT3 sodium channel mutations expressed in human cells and compares mexiletine block of WT and LQT3 mutant $\mathrm{Na}^{+}$channels. In these experiments, voltage clamp pulses were used to repetitively activate WT or mutant $\mathrm{Na}^{+}$channels. Pulses were delivered every $5 \mathrm{~s}$, and the ensemble average and variance was calculated. The variance measurement is a sensitive measure of gating and block (28, $35)$. Fig. 1, $A-D$ show the sodium current measured during voltage clamp steps to $-20 \mathrm{mV}$. Fig. $1, E-H$ shows the ensemble variances from WT and each of the mutant channels. The concentration of mexiletine used was $10 \mu \mathrm{M}$ in all cases. The level of the peak sodium current before and during drug exposure in Fig. 1 is indicated by arrows. Note that in all cases the peak current is reduced less than the late current. The late gating activity and its suppression in the mutant channels is obvious in the figure panels showing the ensemble variances. Note that in WT channels, the variance is near 0 after $20 \mathrm{~ms}$. The mutant channels continue to gate (nonzero variance) during the entire $100 \mathrm{~ms}$ depolarization. In all cases the late gating activity or variance of the mutant channels is greatly suppressed by mexiletine.

Concentration-inhibition relationships of LQT mutant channels. To further probe the relative sensitivity of the late current compared to the peak inward sodium current in each mutant, we investigated concentration-response relationships (Fig. 2). Concentration-inhibition relationships were constructed by comparing peak and late $(100 \mathrm{~ms})$ sodium current suppression by mexiletine. Because we were unable to measure appreciable late current in WT, only suppression of peak currents was analyzed. Remarkably, the late current showed a much greater sensitivity to inhibition by mexiletine. The $\mathrm{IC}_{50}$ concentrations for inhibition of the late current obtained by fitting a binding isotherm to the averaged data (see Eq. 2; Fig. 2, curves) were ( $\Delta \mathrm{KPQ}) 2.1 \mu \mathrm{M}$, (R1644H) $2.9 \mu \mathrm{M}$, and (N1325S)
A

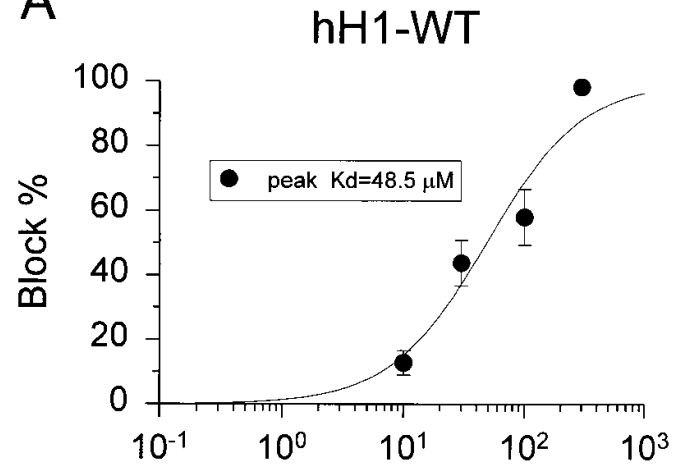

C

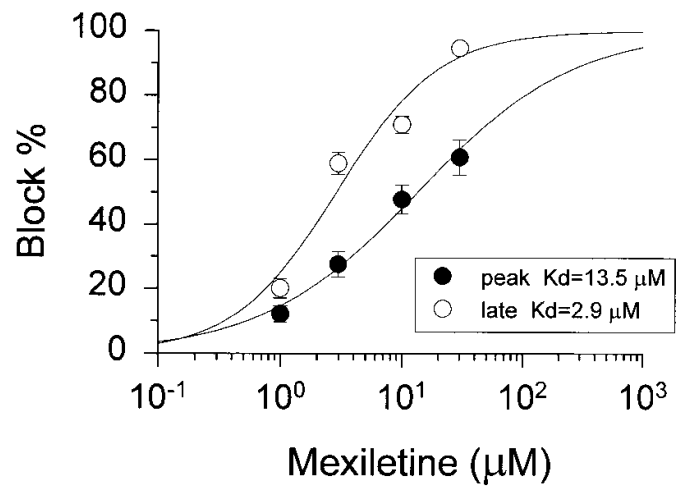

B

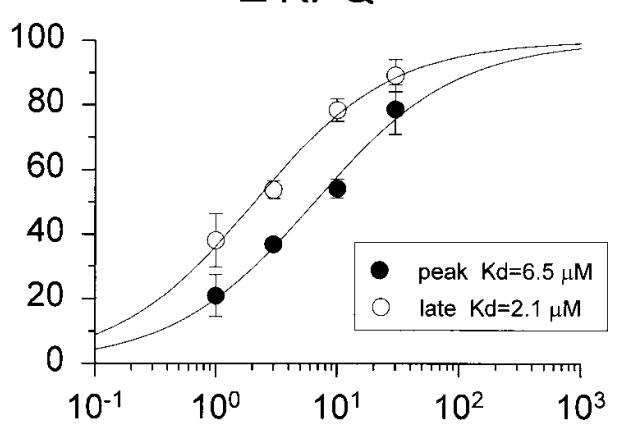

$\mathrm{D}$

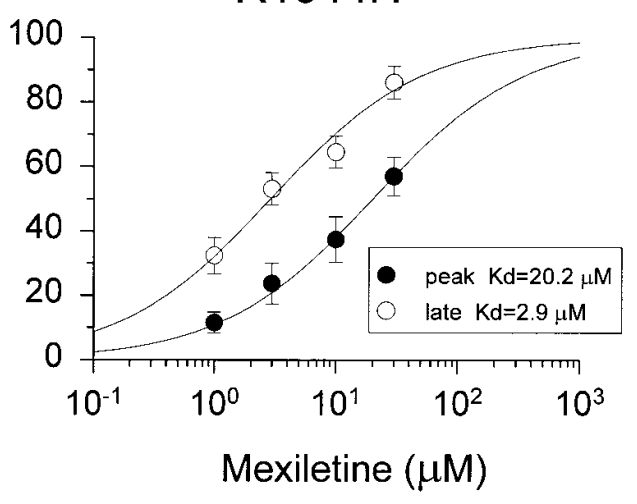

Figure 2. Concentration response curves of peak and late $\mathrm{Na}^{+}$current during a single voltage clamp step for WT $(A), \Delta \mathrm{KPQ}(B), \mathrm{N} 1325 \mathrm{~S}$ $(C)$, and R1644H (D). Cells were voltage clamped to $-120 \mathrm{mV}$ for at least $1 \mathrm{~min}$ to ensure complete removal of inactivation. The membrane potential was then stepped to $-20 \mathrm{mV}$ for $100 \mathrm{~ms}$ to assess the peak inward $\mathrm{Na}^{+}$current and the late opening $\mathrm{Na}^{+}$current in each LQT mutant. Only the inhibition of peak $\mathrm{Na}^{+}$current is shown for the WT channels as no late-opening channels were detected. (Symbols and error bars represent means and SEM. In some cases error bars are smaller than the symbol size.) The lines represent fits of a binding isotherm to data averaged from 3-10 cells each for mutant and WT channels. Apparent $K_{\mathrm{D}}$ values were estimated from these fits to the averaged data. 
$2.9 \mu \mathrm{M}$. In contrast, the $\mathrm{IC}_{50}$ concentrations for inhibition of the peak current obtained by fitting the averaged data were (WT) $48.5 \mu \mathrm{m}(\Delta \mathrm{KPQ}) 6.5 \mu \mathrm{M}$, (R1644H) $20 \mu \mathrm{M}$, and (N1325S) $13.5 \mu \mathrm{M}$. Thus, the sensitivity to inhibition (as reflected in the apparent $K_{\mathrm{D}}$ ) of the peak sodium current was approximately two to eightfold greater in the mutant channels than in WT channels. The sensitivity of the late current was $\sim 20$-fold greater than that of the peak WT current, and the sensitivity of the late current was threefold $(\Delta \mathrm{KPQ}), 6.9$-fold (R1644H), and 4.6-fold (N1325S) greater than that of the peak current in the same mutant.

Targeted suppression does not involve inactivated channels. We tested for mexiletine interactions with the channelinactivated state by a kinetic analysis of inhibition. Channels were driven into the inactivated state by a voltage clamp prepulse and the time course of inactivated-state block development was measured. The time course of block was estimated by the dual pulse protocol described in Fig. $3 \mathrm{~B}$. The ratios of sodium current in the presence of mexiletine divided by the corresponding paired control current are plotted for WT and each mutant (Fig. 3, $A-D$ ). The solid curves in Fig. 3 represent the fitted time course from a model based on binding to a single site. The time constant of block development was de- creased with increasing concentrations of mexiletine both in WT and in the mutants consistent with a first-order binding to the inactivated state of the channel. The time constants for interaction with the inactivated state in the presence of $10 \mu \mathrm{M}$ mexiletine obtained from fitting the averaged data were all $\sim 0.5 \mathrm{~s}$. This time course of binding to inactivated channels can be contrasted to the inhibition of late-opening channels which is fully established within $20 \mathrm{~ms}$ (Fig. 1). Such a slow inhibition of the inactivated state and the rapid establishment of block of the late openings suggest that inactivated-state inhibition is not the mechanism for suppression of the late-opening channels.

The apparent equilibrium dissociation constants for inhibition of channels were calculated from Eq. 3 based on steadystate suppression of the current. The estimated $K_{\mathrm{D}}$ values were: WT, $n=7,14.7 \pm 0.3 \mu \mathrm{M} ; \Delta \mathrm{KPQ}, n=10,21.6 \pm 1.2 \mu \mathrm{M}$; $\mathrm{R} 1644 \mathrm{H}, n=5,12 \pm 2.6 \mu \mathrm{M}$; and N1325S, $n=9,13.8 \pm 1.0 \mu \mathrm{M}$. That these are reasonable estimates is apparent from Fig. 3. Mexiletine $(10 \mu \mathrm{M})$ produced $\sim 50 \%$ suppression in each channel except in the $\Delta \mathrm{KPQ}$ mutant channel where $10 \mu \mathrm{M}$ produced a $30 \%$ suppression on average consistent with its somewhat greater apparent $K_{\mathrm{D}}$. Nevertheless, these data indicate that the mutant channels show approximately (within a factor of two) the same sensitivity to inactivated state block by
A

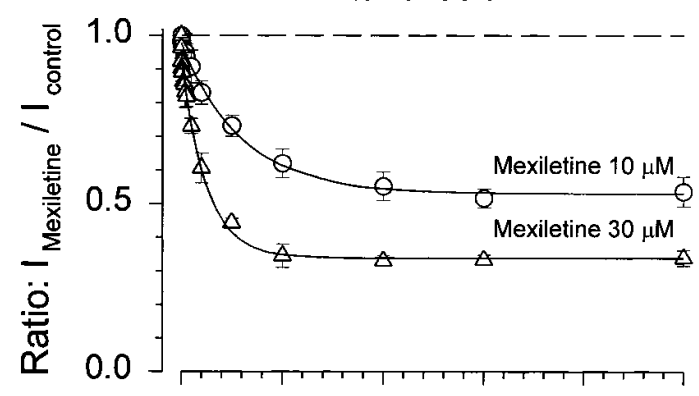

C

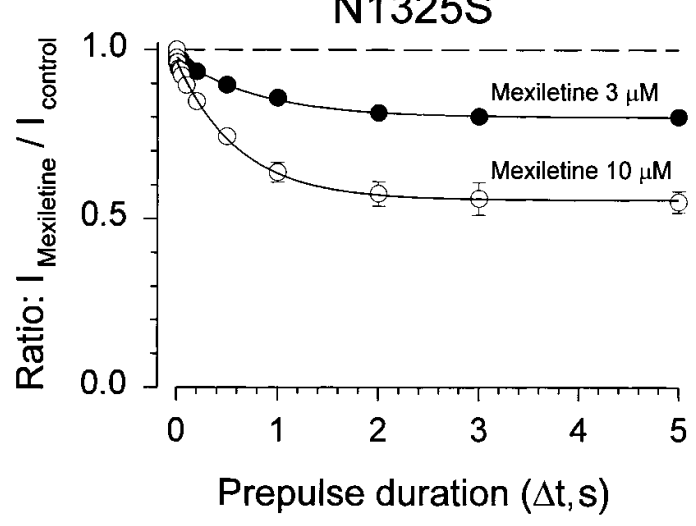

B

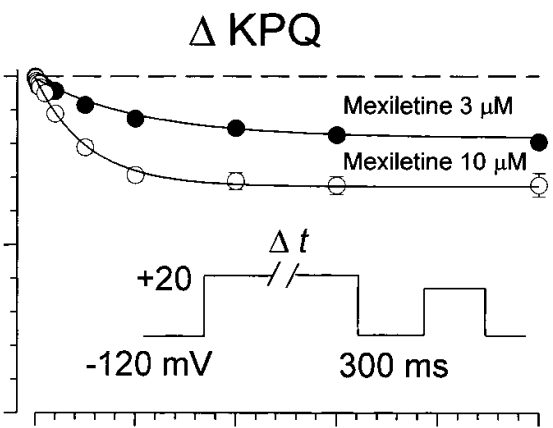

D

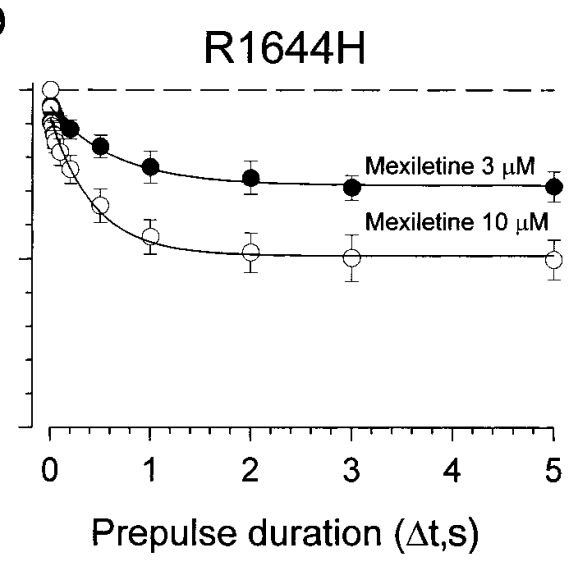

Figure 3. Time course of inactivated state block of $\mathrm{Na}^{+}$ current in WT $(A)$ and in LQT mutant channels $(B-D)$. The mutant channel studied is indicated in each panel. The time course of block development was assessed using a twin-pulse protocol (inset). The duration of the first pulse to $+20 \mathrm{mV}$ was systematically changed to determine the time course of interaction of the inactivated state with mexiletine. The fraction of unblocked channels was measured by a test pulse to $-20 \mathrm{mV}$ that came after a brief period at $-120 \mathrm{mV}$ to allow unblocked, inactivated channels to recover from inactivation. The normalized sodium current in control or in the presence of two different mexiletine concentrations was measured. For the WT channels, 10 and $30 \mu \mathrm{M}$ mexiletine were used. For the mutant channels, 3 and $10 \mu \mathrm{M}$ mexiletine were

used. The ratios of normalized sodium current paired in each cell before and during mexiletine are shown. This transform was used to control for the development of slow inactivation $(\tau=3-6 \mathrm{~s})$. The development of slow inactivation may interfere with an accurate determination of the binding affinity of the fast inactivated state, however any systematic errors in the affinity $\left(K_{\mathrm{D}}\right)$ will be the same for WT and the mutants. (Solid lines) Best nonlinear least square fits of an exponential function. The time constants for block estimated from fitting the averaged WT data in 10 and $30 \mu \mathrm{M}$ were 593 and $245 \mathrm{~ms}$, respectively. The time constants for block estimated from fitting the averaged $\Delta \mathrm{KPQ}$ data in 3 and $10 \mu \mathrm{M}$ were 1,076 and $510 \mathrm{~ms}$, respectively. The time constants for block estimated from fitting the averaged R1644H data in 3 and $10 \mu \mathrm{M}$ were 640 and 433 $\mathrm{ms}$, respectively. The time constants for block estimated from fitting the averaged N1325S data in 3 and $10 \mu \mathrm{M}$ were 807 and $605 \mathrm{~ms}$, respectively. All channels recovered from inactivation within $300 \mathrm{~ms}$. (9) Symbols represent means. Error bars indicate SEM and the lines represent fits to data averaged from 6-10 cells. 
A

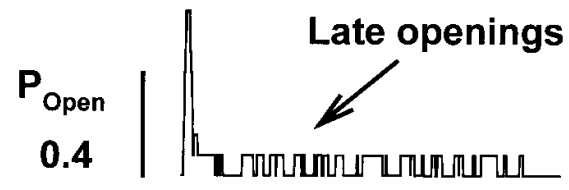

$10 \mathrm{~ms}$

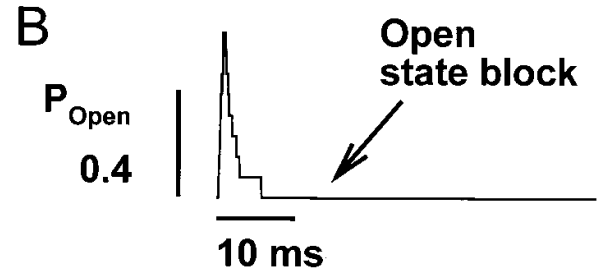

C
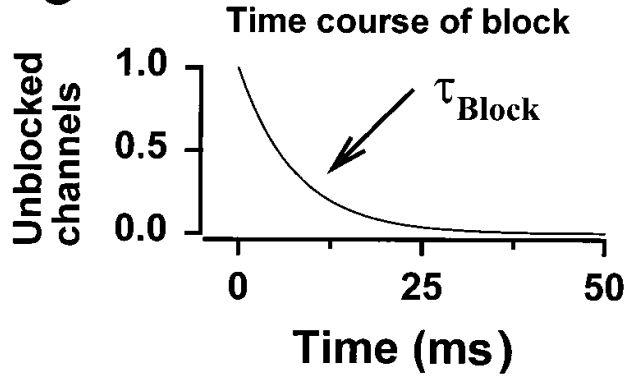

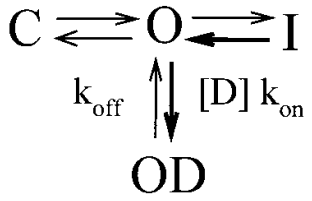

$$
\begin{aligned}
\tau_{\text {Block }} & \simeq\left\{[D] k_{\text {on }}+k_{\text {off }}\right\}^{-1} \\
k_{\text {on }} & =1 \times 10^{7} \mathrm{M}^{-1} \mathrm{~s}^{-1} \\
k_{\text {off }} & =30 \mathrm{~s}^{-1}
\end{aligned}
$$

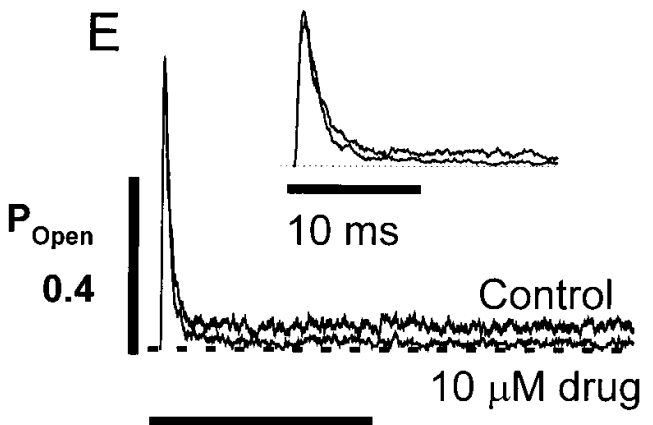

$50 \mathrm{~ms}$
Figure 4. Simulations of an ideal drug-blocking lateopening LQT sodium channels. A linear eight state Markov gating model was used to simulate sodium channel gating and block (7, 35; not shown). Five closed states preceded the open state. The forward rate constants in the activation pathway were $(4 \alpha, 3 \alpha, 2 \alpha, \alpha$, and $\kappa) . \alpha$ and $\kappa$ were set to 10,000 and $5,000 \mathrm{~s}^{-1}$, respectively. The backward rate constants in the activation pathway were $(4 \beta, 3 \beta, 2 \beta, \beta$, and $\lambda$ ). $\beta$ and $\lambda$ were both set to $100 \mathrm{~s}^{-1}$. A single open and inactivated state were used. Channels were placed into the closed state farthest from the open state to simulate resting channels at a negative membrane potential. Rate constants and modal gating were implemented as previously described (7). (A) Gating of channels in the absence of drug. (B) Effect on late openings of a drug

whose time course of block is shown in $C$. An ideal drug has kinetics such that no suppression of peak sodium current occurs but marked suppression of the late openings is achieved. Only the last closed state, the open state, the inactivated state, and the drug-blocked state are shown $(D)$. Drug block was simulated using an association rate constant $\left(k_{\text {on }}\right)$ of $1 \times 10^{7} \mathrm{M}^{-1} \mathrm{~s}^{-1}$. The dissociation rate, 1 , was $30 \mathrm{~s}^{-1}$. The drug concentration was set to $10 \mu \mathrm{M}$ which gives an effective first-order rate constant of entering the blocked state of $100 \mathrm{~s}^{-1}$. Under these conditions the theoretical equilibrium dissociation constant $\left(K_{\mathrm{D}}\right)$ for open-state block was $3 \mu \mathrm{M}$ and the time constant for block (see Eq. 4) development was $\sim 7.7$ ms. Simulations with a smaller off rate (e.g., $10 \mathrm{~s}^{-1}$ ) caused suppression of the peak sodium current as well as the late current similar to our data.

mexiletine as the WT channels. Therefore, inactivated-state block cannot provide selective inhibition of the late-opening channels.

\section{Discussion}

Our data show that all three LQT3 mutant sodium channels are exquisitely sensitive to mexiletine. The inhibition induced by mexiletine is much greater than in WT channels. The inhibition of the LQT3 characteristic late-opening channels was much greater than the inhibition of peak current in both WT and mutant channels. Remarkably the mechanism does not appear to involve the inactivated states of the channel. This was surprising because inactivated-state binding is the mechanism of inhibition of WT channels by mexiletine $(25,26,31,36)$. The therapeutic plasma concentration range for mexiletine is 4-11 $\mu \mathrm{M}$. The mutant channels are significantly inhibited in a tonic fashion by very low concentrations of mexiletine (1-5 $\mu \mathrm{M})$ compared to WT channels (apparent $K_{\mathrm{D}}=49 \mu \mathrm{M}$; see Fig. 2). These concentrations are at the lower end of those that target the inactivated state of WT channels and provide an added measure of selectivity for the mutant channels. An et al. (11) demonstrated selective suppression of the $\triangle \mathrm{KPQ}$ mutant channel by $100 \mu \mathrm{M}$ lidocaine. This was a significant finding but left open the question whether the effect could be achieved with lower, therapeutically relevant concentations of an orally available agent. Furthermore, their study did not address whether the other two mutant channels could be targeted. Our results are significant in light of the recent report by Dumaine et al. (8) who suggest multiple mechanisms for the defects of these mutants. Our data show that the sodium channel LQT defect can be effectively targeted by very low concentrations of drug regardless of the mutation.

Targeted suppression of open LQT channels. A dominant feature of the LQT3 channels is their propensity to more frequently reenter the open state. It is possible that the mutant channels enter a conformation during the altered gating mode that has a higher affinity for mexiletine. We speculated that the dominant-charged species (99\%) of mexiletine opportunistically interacts and inhibits the late, continued openings. An agent that targets open channels could selectively inhibit lateopening mutant channels without greatly modifying the peakopening sodium channels. This would occur because WT sodium channels typically activate and inactivate within $\sim 10 \mathrm{~ms}$ leaving little opportunity for an agent to interact with the transient open state. We explored this hypothesis using the simulations shown in Fig. 4. Here we have used a multistate Markovgating model to simulate sodium channel gating and block (35). Channels were first placed into the closed state farthest from the open state to simulate resting channels at a negative 

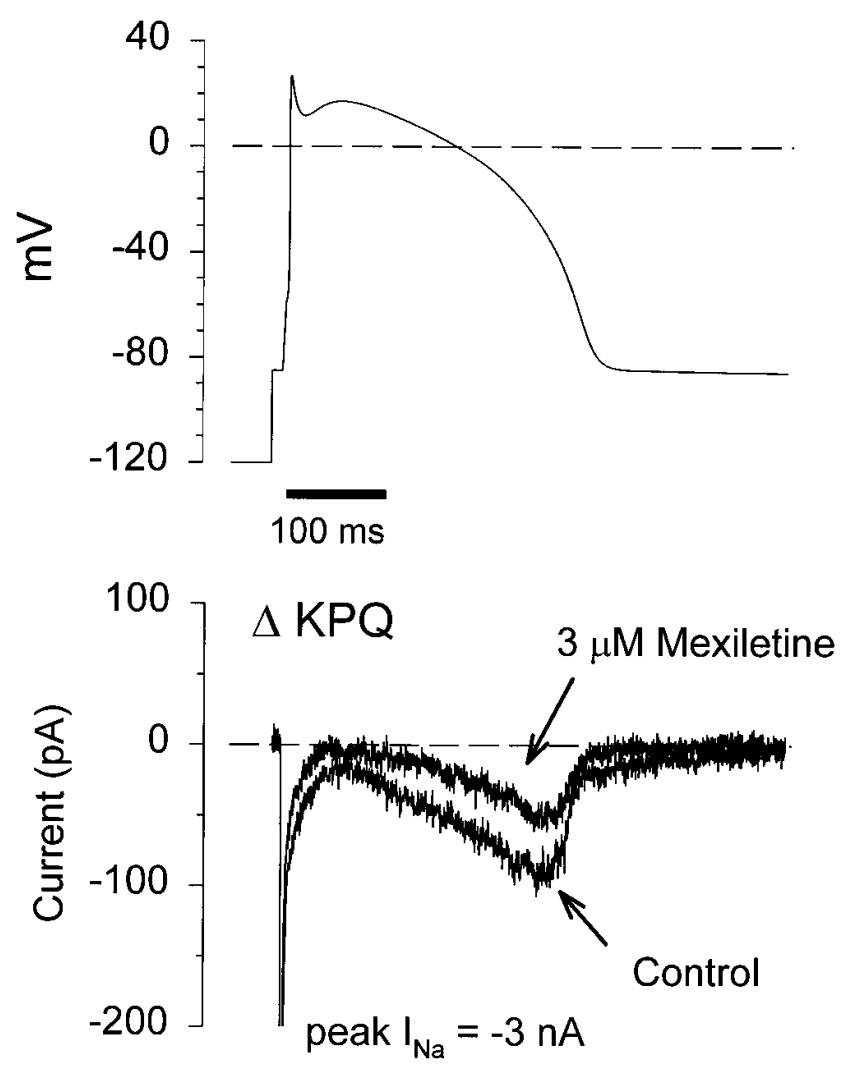

Figure 5. Action potential clamp of a cell expressing the $\triangle \mathrm{KPQ}$ mutation before and during exposure to $3 \mu \mathrm{M}$ mexiletine. The action potential waveform was generated by computer using the Beeler-Reuter action potential model. This waveform was then used, instead of a squarewave step, as a voltage clamp waveform to control the cell membrane potential. (Top) Voltage clamp potential as a function of time. (Bottom) Sodium current that results from clamping the cell to these membrane potentials. Superimposed on the control sodium current tracing is a tracing recorded in the presence of $3 \mu \mathrm{M}$ mexiletine. This concentration suppressed the late sodium current by $\sim 50 \%$ (see Fig. 2).

membrane potential. The rate constants governing transitions among the different conformational states are given in the legend of Fig. 4. The opening of channels in a simulated patch containing five channels is shown in Fig. 4, $A$ and $B$. The time course of drug binding to the open state is shown in Fig. 4 . Ensemble averages of single-channel gating from 750 simulated channels are depicted in Fig. 4 E. The inset of Fig. $4 E$ shows the first $20 \mathrm{~ms}$ on an expanded time scale. Note that with this hypothetical ideal drug the peak sodium current is not reduced but the late current is markedly decreased. Typically the mutant channels undergo transitions into a gating mode which lead to defective inactivation $\sim 5 \%$ of the time or less $(7,8)$. Exposure to $10 \mu \mathrm{M}$ of the drug suppresses these late-opening channels as seen in Fig. 4. The kinetics of interaction with the open state are such that the normally gating channels are not affected, demonstrating that the aberrant-gating mutant channels can be selectively suppressed. These simulations of an ideal compound as an open-state blocker do not fully explain the effects of mexiletine on LQT3 channels. Mexiletine also greatly suppressed the peak sodium current, much more than in the WT channels, suggesting additional effects of the drug on the channels. Our data do not unequivocally demonstrate that open-channel block by mexiletine is the mechanism for the selective suppression of the late openings. Single-channel analysis will be needed to directly test this idea. Nevertheless, this open-state block mechanism would maximize efficacy and minimize nonselective action against normally gating channels. Both WT and mutant channels inactivate and as such little selectivity is afforded by targeting the inactivated state. Only the mutant channels repeatedly reopen allowing them to be targeted. An important goal is to try and identify agents that act by this mechanism.

We also tested whether such low concentrations of mexiletine could significantly impact on late sodium current that would modulate action potential duration in LQT3 by conducting experiments as shown in Fig. 5. We created a computer-generated action potential and used it as the voltage clamp waveform, forcing the membrane potential to follow this action potential trajectory. The sodium current that results is shown below the voltage clamp protocol. During the initial upstroke of the artificial action potential, a large (offscale) inward sodium current occurs. This subsides as the membrane potential approaches the equilibrium potential for sodium ions $(+60 \mathrm{mV})$ reducing the driving force for sodium current. However, the $\triangle \mathrm{KPQ}$ mutant channels continue to open and close. As the membrane repolarizes current through these channels can be seen. In an unclamped cell this late current would have the effect of prolonging the action potential. This late sodium current is suppressed greatly by $3 \mu \mathrm{M}$ mexiletine.

These observations have implications for therapy of LQT3 patients (10). Our data suggest that not only is the late sodium current preferentially suppressed by mexiletine compared to the peak sodium current, the mutant channels in general are selectively targeted by this compound. Furthermore, the high affinity for the defective late opening channels $\left(K_{\mathrm{D}} \sim 3 \mu \mathrm{M}\right)$ compared to the inactivated or rested state affinities of the WT channels $\left(K_{\mathrm{D}}=15-50 \mu \mathrm{M}\right)$ allows suppression of the defective channels at drug concentrations lower than would be used to alter conduction normally $(\sim 3-11 \mu \mathrm{M})$. This selectivity provides an additional margin of safety when using these agents.

\section{Acknowledgments}

This work was supported by grants HL-51197, HL-46681, and NS32387 from the National Institutes of Health, and a Grant-In-Aid from the American Heart Association (Tennessee Affiliate). A.L. George is a Lucille P. Markey Scholar. P.B. Bennett is an Established Investigator of the American Heart Association.

\section{References}

1. Schwartz, P.J., M. Periti, and A. Malliani. 1975. The long QT syndrome. Am. Heart J. 89:378-390.

2. Towbin, J.A., H. Li, R.T. Taggart, M.H. Lehmann, P.J. Schwartz, C.A. Satler, R. Ayyagari, J.L. Robinson, A.J. Moss, and J.F. Hejtmancik. 1994. Evidence of genetic heterogeneity in Romano-Ward long QT syndrome: analysis of 23 families. Circulation. 90:2635-2644.

3. Gellens, M.E., A.L. George, L. Chen, M. Chahine, R. Horn, R.L. Barchi, and R.G. Kallen. 1992. Primary structure and functional expression of the human cardiac tetrodotoxin-insensitive voltage dependent sodium channel. Proc. Natl. Acad. Sci. USA. 89:554-558.

4. George, A.L., T.A. Varkony, H.A. Drabkin, J. Han, J.F. Knops, W.H. Finley, G.B. Brown, D.C. Ward, and M. Haas. 1995. Assignment of the human heart tetrodotoxin-resistant voltage-gated $\mathrm{Na}^{+}$channel $\alpha$ subunit gene (SCN5A) to band 3p31. Cytogenet. Cell Genet. 68:67-70.

5. Wang, Q., J. Shen, I. Splawski, D. Atkinson, Z. Li, J.L. Robinson, A.J. 
Moss, J.A. Towbin, and M.T. Keating. 1995. SCN5A mutations associated with an inherited cardiac arrhythmia, long QT syndrome. Cell. 80:805-811.

6. Wang, O., J. Shen, Z. Li, K. Timothy, G.M. Vincent, S.G. Priori, P.J. Schwartz, and M.T. Keating. 1995. Cardiac sodium channel mutations in patients with long QT syndrome, an inherited cardiac arrhythmia. Hum. Mol. Genet. 4:1603-1607.

7. Bennett, P.B., K. Yazawa, N. Makita, and A.L. George. 1995. Molecular mechanism for an inherited cardiac arrhythmia. Nature (Lond.). 376:683-685.

8. Dumaine, R., Q. Wang, M.T. Keating, H.A. Hartmann, P.J. Schwartz, A.M. Brown, and G.E. Kirsch. 1996. Multiple mechanisms of $\mathrm{Na}^{+}$channel linked long-QT syndrome. Circ. Res. 78:916-924.

9. Wang, D.W., K. Yazawa, A.L. George, and P.B. Bennett. 1996. Characterization of human cardiac $\mathrm{Na}^{+}$channel mutations in the congenital long QT syndrome. Proc. Natl. Acad. Sci. USA. 93:13200-13205.

10. Schwartz, P.J., S.G. Priori, E.H. Locati, C. Napolitano, F. Cantu, J.A. Towbin, M.T. Keating, H. Hammoude, A.M. Brown, L.K. Chen, et al. 1995. Long QT syndrome patients with mutations of the SCN5A and HERG genes have differential responses to $\mathrm{Na}^{+}$channel blockade and to increases in heart rate. Circulation. 92:3381-3386.

11. An, R.-H., S.Z. Bangalore, and R.S. Kass. 1996. Lidocaine block of LQT-3 mutant human $\mathrm{Na}^{+}$channels. Circ. Res. 79:103-108.

12. Bean, B.P., C.J. Cohen, and R.W. Tsien. 1983. Lidocaine block of cardiac sodium channels. J. Gen. Physiol. 81:613-642.

13. Bennett, P.B., C. Valenzuela, L.Q. Chen, and R.G. Kallen. 1995. Molecular basis of lidocaine block of human cardiac $\mathrm{Na}^{+}$channels: mutagenesis of the alpha subunit III-IV interdomain. Circ. Res. 77:584-592.

14. Strichartz, G.R. 1973. The inhibition of sodium currents in myelinated nerve by quaternary derivatives of lidocaine. J. Gen. Physiol. 62:37-57.

15. Schwarz, W., P.T. Palade, and B. Hille. 1977. Local anesthetics. Effect of $\mathrm{pH}$ on use-dependent block of sodium channels in frog muscle. Biophys. J. 20: 343-368.

16. Hille, B. 1977. Local anesthetics: hydrophilic and hydrophobic pathways for the drug-receptor reaction. J. Gen. Physiol. 69:497-515.

17. Hille, B. 1977. The pH-dependent rate of action of local anesthetics on the node of Ranvier. J. Gen. Physiol. 69:475-496.

18. Hondeghem, L.M., and B.G. Katzung. 1977. Time- and voltage-dependent interactions of antiarrhythmic drugs with cardiac sodium channels. Biochim. Biophys. Acta. 472:373-398.

19. Sanchez-Chapula, J., Y. Tsuda, and I.R. Josephson. 1983. Voltage- and use-dependent effects of lidocaine on sodium current in rat single ventricular cells. Circ. Res. 52:557-565.

20. Hondeghem, L.M., and B.G. Katzung. 1984. Antiarrhythmic agents: the modulated receptor mechanism of action of sodium and calcium channel-blocking drugs. Annu. Rev. Pharmacol. Toxicol. 24:387-423.

21. Clarkson, C.W., C.H. Follmer, R.E. Ten Eick, L.M. Hondeghem, and
J.Z. Yeh. 1988. Evidence for two components of sodium channel block by lidocaine in isolated cardiac myocytes. Circ. Res. 63:869-878.

22. Grant, A.O., M.A. Dietz, F.R. Gilliam III, and C.F. Starmer. 1989. Blockade of cardiac sodium channels by lidocaine. Single-channel analysis. Circ. Res. 65:1247-1262.

23. Gilliam, F.R., III, C.F. Starmer, and A.O. Grant. 1989. Blockade of rabbit atrial sodium channels by lidocaine. Characterization of continuous and frequency-dependent blocking. Circ. Res. 65:723-739.

24. Ragsdale, D.S., J.C. McPhee, T. Scheuer, and W.A. Catterall. 1994. Molecular determinants of state-dependent block of $\mathrm{Na}^{+}$channels by local anesthetics. Science (Wash. DC). 265:1724-1728.

25. Ono, M., A. Sunami, T. Sawanobori, and M. Hiraoka. 1994. External $\mathrm{pH}$ modifies $\mathrm{Na}^{+}$channel block by mexiletine in guinea pig ventricular myocytes. Cardiovasc. Res. 28:973-979.

26. Ono, M., A. Sunami, and M. Hiraoka. 1995. Interaction between external $\mathrm{Na}^{+}$and mexiletine on $\mathrm{Na}^{+}$channel in guinea pig ventricular myocytes Pflug. Arch. Eur. J. Physiol. 431:101-109.

27. Wang, D.W., L. Nie, A.L. George, and P.B. Bennett. 1996. Distinct local anesthetic affinities in human muscle $\mathrm{Na}^{+}$channels. Biophys. J. 70:1700-1708.

28. Sigworth, F.J. 1980. The conductance of sodium channels under conditions of reduced current at the node of Ranvier. J. Physiol. (Lond.). 307:131142 .

29. Aldrich, R.W., D.P. Corey, and C.F. Stevens. 1983. A reinterpretation of mammalian sodium channel gating based on single channel recording. $\mathrm{Na}$ ture (Lond.). 306:436-441.

30. Fenwick, E.M., A. Marty, and E. Neher. 1982. Sodium and calcium channels in bovine chromaffin cells. J. Physiol. (Lond.). 331:599-635.

31. Sunami, A., Z. Fan, T. Sawanobori, and M. Hiraoka. 1993. Use-dependent block by mexiletine of $\mathrm{Na}^{+}$currents at the single channel level in guinea pig ventricular myocytes. Br. J. Pharmacol. 110:183-192.

32. Valenzuela, C., and P.B. Bennett. 1994. Gating of $\mathrm{Na}^{+}$channels in excised membrane patches after modification by alpha-chymotrypsin. Biophys. J. 67:161-171.

33. Wang, D.W., A.L. George, and P.B. Bennett. 1996. Comparison of heterologously expressed human cardiac and skeletal muscle $\mathrm{Na}^{+}$channels. Biophys. J. 70:238-245.

34. Hamill, O.P., A. Marty, E. Neher, B. Sakmann, and F.J. Sigworth. 1981 Improved patch-clamp techniques for high-resolution current recording from cells and cell-free membrane patches. Pflug. Arch. Eur. J. Physiol. 391:85-100.

35. Hille, B. 1992. Ionic Channels of Excitable Membranes. 2nd ed. Sinauer Associates, Inc., Sunderland, MA.

36. Yatani, A., and N. Akaike. 1985. Blockade of the sodium current in isolated single cells from rat ventricle with mexiletine and disopyramide. J. Mol. Cell. Cardiol. 17:467-476. 